\title{
Adsorption and Photocatalytic Properties of Modified Rectorite-Titanium Dioxide Composites
}

\author{
Yuanyuan $\mathrm{Du}^{1 *}$, Xinjun $\mathrm{Li}^{1 *}$, Ying $\mathrm{Fu}^{1}$, Linlin Zheng ${ }^{1}$, Xing $\mathrm{Gao}^{1}$, Wen $\mathrm{He}^{1}$ and Pengwu Zheng ${ }^{2}$ \\ ${ }^{1}$ School of Chemical and Biological Engineering, Qilu Institute of Technology, Jinan Shandong. 250200. China \\ ${ }^{2}$ School of Pharmacy, Jiangxi Science and Technology Normal University, Nanchang Jiangxi. 330013. China \\ Yuanyuan $\mathrm{Du}^{1 *}$ and Xinjun $\mathrm{Li}^{1 *}$ contributed equally to this work.
}

\begin{abstract}
Using rectorite as raw material, REC is modified by nitric acid, $\mathrm{TiO}_{2}$ is attached to the surface of the acidized modified REC tablet to form modified REC-TiO 2 composite materials by the sol-gel method, and its adsorption performance and photocatalytic properties were studied. The results show that the adsorption of REC, modified REC, modified REC-TiO 2 composite materialtoporads to methylene blue increased with the increase of equilibrium concentration. The process of adsorption $\mathrm{MB}$ of modified REC-TiO ${ }_{2}$ composite materials is an endothermic process. The adsorption of REC, modified REC, modified REC-TiO 2 composite materials to MB increased with the increase of temperature. When the temperature is 25 degree, the maximum adsorption capacity of REC is $93.985 \mathrm{mg} / \mathrm{g}$, the maximum adsorption capacity of modified REC is $107.006 \mathrm{mg} / \mathrm{g}$, and the maximum adsorption capacity of modified $\mathrm{REC}-\mathrm{TiO}_{2}$ is 120.773 $\mathrm{mg} / \mathrm{g}$. The degradation rate of $\mathrm{MB}$ and p-nitrophenol by modified $\mathrm{REC}-\mathrm{TiO}_{2}$ composite under illumination are $99 \%$ and $98 \%$, respectively. The decontamination rate of $\mathrm{MB}$ and $4-\mathrm{NP}$ of modified $\mathrm{REC}-\mathrm{TiO}_{2}$ composites under darkness was $50 \%$ and $0.8 \%$, respectively. Under light conditions, the degradation rate of $\mathrm{MB}$ and 4-NP in modified REC- $\mathrm{TiO}_{2}$ composite materials is improved.
\end{abstract}

\section{Introduction}

At present, the wanton discharge of agricultural and industrial wastewater has caused serious environmental pollution, which has aroused widespread concern of the society.

The REC and montmorillonite are deposited alternately as one layer. It itself is a good adsorbent, but the adsorption capacity is limited. It can be greatly improved by some chemical modification. It has a very wide application and good development prospects in environmental protection.

As a promising semiconductor photocatalytic material, $\mathrm{TiO}_{2}$ is widely used in various fields due to its good biochemical characteristics, high catalytic activity and good oxidation performance, which is bound to lead the new direction of environmental protection industry. Therefore, the modified rec composite can be prepared on the basis of acidification modified REC, so that the prepared composite has good adsorption performance and photocatalytic performance.

\section{Experimental methods}

\subsection{Preparation of modified REC- $\mathrm{TiO}_{2}$ composite}

\subsubsection{Preparation of modified REC}

Weigh $10.00 \mathrm{~g}$ REC with an electronic balance, put it into a $250 \mathrm{~mL}$ beaker, add $100 \mathrm{~mL} 3 \mathrm{~mol} / \mathrm{L}$ nitric acid for pickling, put it into a $100{ }^{\circ} \mathrm{C}$ constant temperature water bath, mechanically stir it for $3 \mathrm{~h}$ with a powerful electric mixer, wash the sample with deionized water and centrifuge it with a low-speed centrifuge, repeat several times until $\mathrm{pH} \approx 7$. The modified rec was dried at $105{ }^{\circ} \mathrm{C}$ for $12 \mathrm{~h}$. The modified rec was obtained by grinding the sample with mortar and passing through 200 mesh sieve.

\subsubsection{Preparation of modified REC-TiO 2 composites by sol-gel method}

Use a measuring cylinder to accurately measure $40.0 \mathrm{~mL}$ absolute ethanol and add it into a $200 \mathrm{ml}$ dry beaker. Accurately measure $15.0 \mathrm{~mL}$ butyl titanate with a measuring cylinder, slowly add it along the beaker wall, and mechanically stir the solution at room temperature for $15 \mathrm{~min}$. Accurately measure $6.0 \mathrm{~mL}$ glacial acetic acid, and slowly drop it into the beaker with a rubber tipped burette (delay the violent hydrolysis of butyl titanate). The obtained solution was stirred vigorously for 15 min with a strong electric mixer until the solution was transparent. Weigh $6.00 \mathrm{~g}$ of modified rec with an electronic balance, add it to the transparent solution, mechanically stir it in a constant temperature water bath

*Corresponding author's email: duyuan01@126.com,928878031@qq.com 
at $30{ }^{\circ} \mathrm{C}$, and react for $1 \mathrm{~h}$ to obtain solution $\mathrm{A}$.

Weigh $3.40 \mathrm{~g}$ ammonium nitrate with an electronic balance, add it into a $200 \mathrm{~mL}$ dry beaker, transfer $12 \mathrm{~mL}$ deionized water and $10 \mathrm{ml}$ absolute ethanol respectively, and prepare solution $\mathrm{B}$.

Slowly add solution B to solution a with a rubber tip dropper and mechanically stir until $\mathrm{pH} \approx 4$. Put the mixture into a constant temperature water bath at $40{ }^{\circ} \mathrm{C}$. After mechanical stirring $0.5 \mathrm{~h}$, the gel was made. The semi-finished products were sealed with fresh-keeping film for $12 \mathrm{~h}$, washed with deionized water and centrifuged at low speed for several times until the $\mathrm{pH}$ was 7. Put the sample into $105{ }^{\circ} \mathrm{C}$ electric blast drying oven, and dry for $12 \mathrm{~h}$ till drying. The modified REC- $\mathrm{TiO}_{2}$ composite was obtained by grinding in a mortar, passing through a 200 mesh sieve, calcining in an intelligent box type high temperature furnace at $300^{\circ} \mathrm{C}$ for $3 \mathrm{~h}[1]$.

\subsection{Adsorption experiment of methylene blue}

\subsubsection{Drawing of standard curve}

Accurately weigh $0.0005 \mathrm{~g}, 0.0010 \mathrm{~g}, 0.0015 \mathrm{~g}, 0.0020 \mathrm{~g}$ and $0.0025 \mathrm{~g} \mathrm{MB}$ solids with an electronic analytical balance, put them into different beakers, dissolve them, transfer them into a $250 \mathrm{~mL}$ volumetric flask with labels, add deionized water to make a constant volume to the scale line, shake them well, and prepare them into 2 $\mathrm{mg} / \mathrm{L}, 4 \mathrm{mg} / \mathrm{L}, 6 \mathrm{mg} / \mathrm{L}, 8 \mathrm{~m} \mathrm{~L}$ and $10 \mathrm{mg} / \mathrm{L}$ The absorbance of MB solution was measured with UV-vis spectrophotometer and recorded. The standard curve of concentration (c) and absorbance (A) of MB solution was drawn.

\subsubsection{Adsorption kinetics}

0.0050 g REC, modified REC and modified REC-TiO2 composite were accurately weighed by electronic analytical balance and added into three different parts of $10.00 \mathrm{~mL} 0.1 \mathrm{mmol} / \mathrm{L} \mathrm{MB}$ solution. Put the oscillator into the water bath at a constant temperature to measure the absorbance, and record the absorbance with the water bath at $25{ }^{\circ} \mathrm{C}$ every 15 minutes. Set the temperature to $35^{\circ} \mathrm{C}$ and $45^{\circ} \mathrm{C}$, and repeat the above operation in turn.

\subsubsection{Adsorption isotherm}

Take $10.00 \mathrm{~mL}$ of MB solution from $0.01 \mathrm{mmol} / \mathrm{L}, 0.05$ $\mathrm{mmol} / \mathrm{L}, 0.1 \mathrm{mmol} / \mathrm{L}, 0.2 \mathrm{mmol} / \mathrm{L}, 0.3 \mathrm{mmol} / \mathrm{L}, 0.4$ $\mathrm{mmol} / \mathrm{L}, 0.5 \mathrm{mmol} / \mathrm{L}, 0.6 \mathrm{mmol} / \mathrm{L}, 0.7 \mathrm{mmol} / \mathrm{L}$ and $0.8 \mathrm{mmol} / \mathrm{L} \mathrm{MB}$ solution respectively with a $10 \mathrm{ml}$ pipette. Take 3 portions of each solution and put them into different labeled glass bottles. $0.0050 \mathrm{~g}$ REC, 10 parts of modified REC and 10 parts of modified REC-TiO ${ }_{2}$ composite were accurately weighed by electronic analytical balance and put into glass bottles with different concentrations of MB solution. The glass bottle was placed in a water bath constant temperature oscillator at $25{ }^{\circ} \mathrm{C}$ and oscillated at constant temperature until the adsorption reached saturation. The absorbance was measured by ultraviolet visible spectrophotometer and recorded.

\subsection{Study on Photocatalytic Performance}

Two parts of $0.0010 \mathrm{~g}$ of modified REC-TiO ${ }_{2}$ composite were accurately weighed with an electronic analytical balance and added into $20 \mathrm{~mL}$ of $0.1 \mathrm{mmol} / \mathrm{L} \mathrm{MB}$ solution respectively, and then shaken up. One was irradiated by $365 \mathrm{~nm}$ triple UV analyzer, and the other was left in the dark. The absorbance was measured every half an hour and recorded. The test was stopped after 4 hours.

Two parts of $0.0020 \mathrm{~g}$ of modified REC-TiO ${ }_{2}$ composite were accurately weighed with an electronic analytical balance and added into $10 \mathrm{~mL}$ of $10 \mathrm{mg} / \mathrm{L}$ 4-NP solution respectively, and then shaken up. One part was irradiated by $253 \mathrm{~nm}$ triple UV analyzer, and the other part was left in the dark. The absorbance was measured every half an hour and recorded. The test was stopped after 4 hours.

\subsection{Infrared spectrum analysis}

REC, modified REC and modified REC-TiO 2 composite materials were mixed with potassium bromide at the ratio of 1:100 in an agate mortar and ground, then pressed with a tablet press, and finally scanned with an infrared spectrometer. The resolution of the infrared spectrometer is $4 \mathrm{~cm}-1$, the analysis range is $400-4000 \mathrm{~cm}^{-1}$, and the accuracy is $0.01 \mathrm{~cm}^{-1}$. Analysis of the wavelength range of each absorption band, infer that there should be some functional groups in the molecule, in order to identify the sample.

\section{Results and discussion}

\subsection{Adsorption experiment of methylene blue}

\subsubsection{Adsorption kinetics}

In order to study the kinetic mechanism of adsorption process, pseudo second order kinetic model is often used to simulate the experimental data [5].According to the pseudo second order kinetic equation: $\frac{t}{q_{t}}=\frac{1}{k q_{e}^{2}}+\frac{t}{q_{e}}$ (1)

Where $\mathrm{k}(\mathrm{mg} / \mathrm{g} \cdot \mathrm{h})$ is the second-order adsorption rate constant [6], $\mathrm{q}_{\mathrm{t}}(\mathrm{mg} / \mathrm{g})$ is the adsorption capacity of $\mathrm{MB}$ at $\mathrm{t}(\mathrm{h}), \mathrm{q}_{\mathrm{e}}(\mathrm{mg} / \mathrm{g})$ is the adsorption capacity of MB at equilibrium time [7]. The kinetic parameters obtained from the experimental data are shown in Table 1 and Table 2. 
Table 1. Second order kinetic parameters of MB adsorption by REC, modified REC and modified REC-TiO 2 composites with different time

\begin{tabular}{cccc}
\hline Sample & $\mathrm{k}(\mathrm{mg} / \mathrm{g} \cdot \mathrm{h})$ & $\mathrm{q}_{\mathrm{e}}(\mathrm{mg} / \mathrm{g})$ & $\mathrm{R}^{2}$ \\
\hline REC & 0.013 & 74.627 & 0.996 \\
Modified & 0.027 & 78.125 & 0.996 \\
$\begin{array}{l}\text { REC } \\
\text { Modified }\end{array}$ & 0.048 & 79.365 & 0.999 \\
$\mathrm{REC}_{\mathrm{TiO}}{ }_{2}$ & & & \\
\hline
\end{tabular}

Table 2. Second order kinetic parameters of MB adsorption by modifiedREC- $\mathrm{TiO}_{2}$ composite at different temperatures

\begin{tabular}{cccc}
\hline $\begin{array}{c}\text { Modified } \\
\mathrm{REC}^{-T i O}{ }_{2}\end{array}$ & $\begin{array}{c}\mathrm{k}(\mathrm{mg} / \mathrm{g} \cdot \\
\mathrm{h})\end{array}$ & $\mathrm{q}_{\mathrm{e}}(\mathrm{mg} / \mathrm{g})$ & $\mathrm{R}^{2}$ \\
\hline $25^{\circ} \mathrm{C}$ & 0.027 & 75.414 & 0.999 \\
$35^{\circ} \mathrm{C}$ & 0.037 & 76.982 & 0.999 \\
$45^{\circ} \mathrm{C}$ & 0.062 & 78.864 & 0.999 \\
\hline
\end{tabular}

\subsection{Study on Photocatalytic Performance}

Under the photocatalysis, $\mathrm{TiO}_{2}$ can degrade $\mathrm{MB}$ by demethylation. At the wavelength of $662 \mathrm{~nm}$, the absorbance of the unabsorbed MB solution decreases. The degradation rate of MB was $99 \%$ in light and $50 \%$ in dark. Under the condition of ultraviolet lamp irradiation, the modified REC-TiO ${ }_{2}$ composite has strong photodegradation effect on $\mathrm{MB}$ in addition to adsorption effect, which makes the degradation rate under light condition higher than that under dark condition.

Under photocatalysis, the modified $\mathrm{REC}-\mathrm{TiO}_{2}$ composite can remove 4-NP, but not affected by its adsorption performance. The degradation rate of 4-NP was $98 \%$ in light and $0.8 \%$ in dark. Under the irradiation of UV lamp, the modified REC- $\mathrm{TiO}_{2}$ composite has a strong photodegradation effect on 4-NP, which makes the degradation rate of 4-NP in the light condition higher than that in the dark.

\subsection{Infrared spectrum analysis}

The Fourier transform infrared spectra of REC, modified REC and modified REC- $\mathrm{TiO}_{2}$ composites were analyzed. The results show that the absorption peaks at $3630 \mathrm{~cm}^{-1}$ and $1640 \mathrm{~cm}^{-1}$ are bending vibration absorption peaks of hydrogen bond formed by interlayer adsorbed water; the absorption peaks at $3390 \mathrm{~cm}^{-1}$ are Tensile vibration absorption peaks of -OH formed by silicon hydroxyl group; the absorption peaks at $1100 \mathrm{~cm}^{-1}$ are bending vibration absorption peaks The absorption peak at 916 $\mathrm{cm}^{-1}$ is the stretching vibration absorption peak of $-\mathrm{OH}$ formed by aluminum hydroxyl group; the absorption peak at $525 \mathrm{~cm}^{-1}$ is the bending vibration absorption peak formed by silicon oxygen bond; the absorption peak at $460 \mathrm{~cm}^{-1}$ is the stretching vibration absorption peak formed by aluminum hydroxyl group The absorption peak at $\mathrm{cm}^{-1}$ is the bending vibration absorption peak formed by the aluminosilicate bond. The results show that the absorption peak at $1390 \mathrm{~cm}^{-1}$ is the vibration absorption peak caused by the reaction of modified rec with nitric acid; the absorption peak at $920 \mathrm{~cm}^{-1}$ is the characteristic absorption peak of $\mathrm{TiO}_{2}$ loaded in the modified $\mathrm{REC}-\mathrm{TiO}_{2}$ composite; when $\mathrm{TiO}_{2}$ particles are loaded on the modified rec sheet, the absorption peak at $3630 \mathrm{~cm}^{-1}$ is the highest The blue shift of the absorption peak at $\mathrm{cm}^{-1}$ indicates that there is a strong interaction between $\mathrm{TiO}_{2}$ particles and modified REC.

\section{Conclusion}

REC was modified by nitric acid and $\mathrm{TiO}_{2}$ was attached to the surface of modified REC by butyl titanate to form modified REC-TiO ${ }_{2}$ composite. The loading of $\mathrm{TiO}_{2}$ particles in the modified REC-TiO ${ }_{2}$ composite makes the adsorption rate of $\mathrm{MB}$ on the modified $\mathrm{REC}-\mathrm{TiO}_{2}$ faster than that on the modified rec and REC. The adsorption capacity of modified REC-TiO ${ }_{2}$ composite for MB at $45{ }^{\circ} \mathrm{C}$ is higher than that at $25^{\circ} \mathrm{C}$ and $35^{\circ} \mathrm{C}$, indicating that the adsorption process of modified $\mathrm{REC}-\mathrm{TiO}_{2}$ composite for $\mathrm{MB}$ is endothermic. The MB adsorption capacity of rec, modified rec and modified $\mathrm{REC}-\mathrm{TiO}_{2}$ increased with the increase of equilibrium concentration. When the temperature was $25{ }^{\circ} \mathrm{C}$, the maximum adsorption capacity of rec was $93.985 \mathrm{mg} / \mathrm{g}$; the maximum adsorption capacity of modified rec was $107.006 \mathrm{mg} / \mathrm{g}$; the maximum adsorption capacity of modified REC-TiO ${ }_{2}$ composite was $120.773 \mathrm{mg} / \mathrm{g}$, indicating that the adsorption performance of modified $\mathrm{REC}^{-\mathrm{TiO}_{2}}$ composite was much higher than that of modified rec and rec original sample. The adsorption capacity of REC, modifiedREC and modified REC- $\mathrm{TiO}_{2}$ composite for MB increased with the increase of temperature, and finally reached the adsorption saturation state. The degradation rates of $\mathrm{MB}$ and 4-NP of the modified REC- $\mathrm{TiO}_{2}$ composite were $99 \%$ and $98 \%$ respectively under light, and $50 \%$ and $0.8 \%$ respectively under dark, which indicated that the light condition was beneficial to improve the photocatalytic activity of the modified REC-TiO ${ }_{2}$ composite.

\section{Reference}

1. Liu Chengdong, Wu Yixin, Meng Xiangyu, et al. Construction of Two Novel Titanium Oxide Clusters: Copper Ion Introducing Enhances Photocatalytic Performance. 2020, 2020(44):4189-4194.

2. Ziqiang Yin, Rique Jie En Yeow, Yunqiao Ma, et al. Link between interfacial interaction and membrane fouling during organic solvent ultrafiltration of colloidal foulants. 2020, 611

3. Masayuki Yamane, Shinji Aso, Teruo Sakaino. Preparation of a gel from metal alkoxide and its properties as a precursor of oxide glass[J]. Journal of Materials Science, 1978, 13(4): 865-870.

4. Jingyi Li, Chuncheng Chen, Jinchai Zhao, et al. 
Photodegradation of dye pollutants on $\mathrm{TiO}_{2}$ nanoparticles dispersed in silicate under UV-Vis irradiation[J]. Appl Catal B, 2002, 37: 331-338.

5. Harstrick A, KutO M, Heinzle E. $\mathrm{TiO}_{2}$-assisted degradation of environmentally relevant organic compounds in waste water using a novel fluidised bed photoreactor[J]. Environmental Science and Technology, 1996, 30: 817-826.

6. Bonamali Pal, Maheshwar Sharon, Gyoichi Nogami. Preparation and characterization of $\mathrm{TiO}_{2} / \mathrm{Fe}_{2} \mathrm{O}_{3}$ binary mixed oxides and its photocatalytic properties[J]. Materials Chemistry and Physics, 1999, 59: 254-261.

7. Jiaguo Yu, Xiujian Zhao, Qingnan Zhao. Effect of surface on photocatalytic activity of $\mathrm{TiO} 2$ thin films prepred by sol-gel method[J]. Thin Solid Films, 2000, 379: 7-14.

8. Hiroshi Yoneyama, Shigeo Haga, Shoji Yamanaka. Photocatalytic Activities of Microcrystalline $\mathrm{TiO}_{2}$ Incorporated in Sheet Silicates of Clay[J]. The Journal of Physical Chemistry, 1989, 93(12): 4833-4837. 